\title{
Magnitude of sex differences in visual search varies with target eccentricity
}

\author{
Michael C. W. English ${ }^{1}$ (D) Murray T. Maybery ${ }^{1} \cdot$ Troy A. W. Visser $^{1}$
}

Published online: 1 September 2020

(C) The Psychonomic Society, Inc. 2020

\begin{abstract}
A recent meta-analysis found no support for the popular theory that superior visuospatial ability in males is attributable to their relatively greater hemispheric asymmetry of neural functions. However, the issue of whether differences in hemispheric laterality could account for differences in visual perception between the sexes has not been systematically investigated. Visual search is an ideal task for such an investigation, as target-position can be systematically varied across the visual field allowing for a detailed analysis of how performance varies with visual field and eccentricity. We recruited 539 undergraduate participants (150 male) and administered a visual search task that required them to identify the presence of a uniquely-oriented triangle amongst distractors. Crucially, target location was systematically varied over the visual field across trials. Males displayed both superior accuracy and shorter reaction time when targets were presented in the left visual field, whilst sex differences systematically diminished when the target was located further rightward. These behavioural results are in line with the notion that greater hemispheric asymmetry in males influences task performance to a varying extent across the visual field, and illustrates the importance of considering task parameters and the influence of sex in behavioural research.
\end{abstract}

Keywords Visual perception $\cdot$ Visual search $\cdot$ Sex differences $\cdot$ Lateralization

\section{Introduction}

Over 40 years ago, Jerre Levy $(1972,1978)$ postulated that sex-based differences in visuospatial and verbal cognitive abilities might be the consequence of differences in the distribution of neural functions across the hemispheres. The theory enjoyed substantial longevity, in part due to independent lines of evidence that somewhat corroborated the account. For one, there is relatively consistent evidence that high-level verbal and visuospatial functions are more asymmetrically distributed across hemispheres in males than females (Heilman, 1995; Hellige, 1993; Ocklenburg \& Güntürkün, 2017). Secondly, despite current evidence indicating relatively more sex similarities than differences in cognitive abilities (Hyde, 2005;

Electronic supplementary material The online version of this article (https://doi.org/10.3758/s13423-020-01796-7) contains supplementary material, which is available to authorized users.

Michael C. W. English

michael.english@uwa.edu.au

1 School of Psychological Science, University of Western Australia, 35 Stirling Highway, Crawley, WA 6009, Australia
Jäncke, 2018; Zell et al., 2015), the differences that exist generally fall into a trend of males and females showing respective advantages on certain spatial and verbal tasks (for a review, see Halpern, 2012). However, a recent meta-analysis of studies combining behavioural measures of cognitive ability with neuroimaging techniques revealed that the predicted pattern of concurrent differences in hemispheric specialization and abilities between men and women is actually rarely obtained (Hirnstein et al., 2018). For example, for male compared to female participants, Hahn et al. (2010) reported stronger hemispheric asymmetry but not superior mental rotation, while Rilea (2008) reported superior mental rotation but not stronger hemispheric asymmetry.

Whilst this meta-analysis dispels the notion that increased hemispheric specialization is correlated with enhanced performance on more complex visuospatial tasks and could distinguish the two sexes, the possibility remains that aspects of visual perception could differ as a function of sex-linked hemispheric specialization. Sex differences in visual perception are observed in numerous tasks including contrast sensitivity, visual acuity, colour perception and motion perception, although the direction of differences is inconsistent (for a review, see Vanston \& Strother, 2017). Furthermore, several 
lines of evidence suggest that alterations in hemispheric asymmetry can influence visual perception - especially with respect to perception across the visual field. The influence of hemispheric asymmetry is perhaps most pronounced in patients with unilateral brain lesions. For example, patients with right-sided lesions over various cortical regions fail to consciously attend to targets presented in the left visual field on the line-cancellation task (Ferber \& Karnath, 2001). This outcome seems to arise from disruptions to perceptual processing (Heilman et al., 2003) as these patients perform comparably to health controls when adapted to prisms that shift information to the right visual field, from which it is projected to the intact left hemisphere (Saevarsson et al., 2009; Vangkilde \& Habekost, 2010).

As well as asymmetries arising from lesions, perception also seems to be affected by hemispheric asymmetries in healthy individuals. For example, most individuals show a small, but reliable, over-attendance or perceptual exaggeration of stimulus features (such as length and shading; Jewell \& McCourt, 2000; Nicholls et al., 1999) presented in the left visual field (LVF). This phenomenon has been termed pseudoneglect (Bowers \& Heilman, 1980) given the similarity to left-neglect observed for patients with right-side unilateral lesions and in recognition that it occurs in the absence of hemispheric damage. The source of this attentional bias appears to be a combination of learned and innate processes. On the one hand, it is suggested that exposure to the English language's left-to-right format influences hemifield biases. For example, studies have shown that the left hemifield advantage for identifying Latin (English) letters in a rapid serial visual presentation is eliminated when Arabic characters are presented instead (Ransley et al., 2018), and changing the implied reading direction by rotating the orientation and position of Latin letters modulates the location in which the advantage is present (Holcombe et al., 2017). On the other hand, comparable LVF biases on the perceptual greyscales task have been observed for both English- and Hebrew-speaking individuals, and eye-tracking studies have demonstrated that infants as young as 11 months prefer to explore the left side of centrally presented faces, well before reading direction could reasonably influence attentional or perceptual biases (Dundas et al., 2012). ${ }^{1}$ This suggests that asymmetric hemispheric organisation where visual processing is dominant in the right hemisphere (for a review, see Verleger \& Śmigasiewicz, 2015) also has a role to play in LVF biases.

A candidate behavioural paradigm to explore lateralised sex differences in perception is the well-established visual search task, in which multiple distractor stimuli are distributed

\footnotetext{
${ }^{1}$ It should be noted that this LVF advantage is not ubiquitous as instances of the opposite patterns (i.e. right visual field advantage) have been observed with some stimuli (e.g. greater left hemisphere activation when matching 'face parts'; Rossion et al., 2000).
}

across the visual field along with a single target. In its simplest form, targets can be distinguished from distractors based on a unique perceptual feature (e.g. a tilted line amongst vertical lines). This yields fast and efficient search regardless of the number of items in the visual field. In the more complex conjunctive search, the target shares perceptual features with the distractors, thus necessitating perceptual processing of multiple stimulus features before a decision is made about each stimulus. As a result, reaction times are generally longer for conjunctive search relative to featural search, as a more serial search is required to complete the conjunctive task (Treisman \& Gelade, 1980; Wolfe, 1994).

With a visual search task, the location of the targets can be systematically manipulated to examine the influence of eccentricity in addition to visual field. This is important because as acuity declines when visual information is presented further from the fovea, the quality of information projected to the respective hemispheres also diminishes (Anstis, 1974). This can result in the degradation of task performance, as seen in Jordan et al. (2003), where accuracy for letter identification was greatest for centrally presented letters and gradually diminished as eccentricity increased. Given the differences in hemispheric lateralisation between males and females, it is possible that the influence of eccentricity on visual perception might also vary by sex. In turn, differences in patterns of task accuracy or reaction time arising from these sex-based differences are more likely to be detected in a visual search task that systematically varies target eccentricity than simpler 'visual half-field' designs that reduce the visual field to a 'left or right' dichotomy (Behrmann et al., 2004; Poynter \& Roberts, 2012).

Despite the obvious advantages conveyed by using a visual search paradigm, to our knowledge, sex differences have been examined only in a handful of studies investigating the effect of target location using conjunctive visual search tasks (Efron et al., 1987, 1990a, 1990b; Yund et al., 1990a, 1990b). Whilst two studies did find that males were more sensitive to targets presented in the LVF than were females (Efron et al., 1987, 1990a), the stimulus displays spanned a relatively small portion of an observer's visual field (furthest stimulus in the array was approximately $5.25^{\circ}-6.42^{\circ}$ from central fixation across these studies), thus limiting the informativeness of these studies on the extent of hemispheric differences. Exacerbating this issue is the lack of clarity in how foveally presented information (typically $<2^{\circ}$ from fixation), which accounted for a substantial proportion of trials in these studies, is processed. The split fovea theory suggests the fovea is cleanly divided in half with information presented left (right) of fixation projected to the right (left) hemisphere, whilst bilateral projection theory suggests that foveal information is projected bilaterally (i.e. to both hemispheres simultaneously; for a review, see Ellis \& Brysbaert, 2010). Lastly, these studies recruited only $30-50$ participants of each sex per study - a relatively small number considering the small effect sizes typically 
found for cognitive sex differences (Hirnstein et al., 2018) thus perhaps accounting for their inconsistent outcomes.

In sum, there is evidence that differences in visual search performance across the hemifields are linked to hemispheric asymmetries, and that the right hemisphere may be driving these asymmetries (Nicholls et al., 2017; Poynter \& Roberts, 2012). It follows then that if sex differences are the result of differences in hemispheric specialization, the magnitude of the difference should be greatest for targets located in the LVF (as they are projected to the right hemisphere) and gradually decline as the target's location is shifted systematically towards the right visual field (RVF; reflecting a gradual decrease in target processing by the right hemisphere). We tested this prediction by recruiting a large number of participants $(\mathrm{N}$ = 539) from a university population who completed a conjunctive-based search task where targets systematically varied in location across the visual field. From the search array centre, a range of eccentricities was tested extending from $0.75^{\circ}$ to $14.27^{\circ}$. We predicted that sex differences on the task would vary depending on the horizontal position of the target in the search array, reflecting the differences in hemispheric asymmetry between males and females. Specifically, any male superiority on the task (measured using accuracy and reaction time) should be largest for targets with the greatest eccentricities in the LVF and smallest (or absent) for targets with the greatest eccentricities in the RVF.

\section{Methods}

\section{Ethical approval}

Approval to conduct the study was received from the Human Research Ethics Office at the University of Western Australia and the study was carried out in accordance with the provisions of the World Medical Association Declaration of Helsinki. All participants provided written informed consent prior to any experimental participation.

\section{Participants}

Participants were a large, convenience sample of students in an undergraduate psychological research methods unit at the University of Western Australia who completed the visual search task as part of classroom activity. Data were analysed from 539 right-handed ${ }^{2}$ students who consented to the use of their anonymised data for research purposes. Twenty-two participants were identified as outliers based on accuracy and reaction-time criteria (described in the Results section) and, following their removal from the dataset, the final sample

\footnotetext{
$\overline{2}$ Right-handedness defined as a score greater than 0.4 on the Edinburgh Handedness Index (Oldfield, 1971).
}

consisted of 149 males and 368 females with a mean age of $22.24(S D=6.35)$ and $21.51(S D=6.25)$ years, respectively.

Despite recruiting a convenience sample, we conducted a power analysis to determine the overall sample size required to detect a 'meaningful' effect size (approx. $d=.40$, or $\eta^{2}=$ .039 (Hattie, 2009)) using $\mathrm{G}^{*}$ Power (version 3.1.9.7) (Faul et al., 2007). Basing the power analysis on an interaction between the two main variables of interest, sex (betweensubjects, two levels) and target position (within-subjects, ten levels), using a power of .80, and assuming a correlation of .50 among the repeated measures (target position), we estimated an overall sample size of 516 was required. Whilst our study had the required number of participants (indeed, 517 remained after removing outliers), it should be noted that participant sex was not evenly distributed, thus meaning that the testing of any effects within each sex would be more powerful for females compared to males.

\section{Materials}

Each participant was seated approximately $500 \mathrm{~mm}$ in front of an 18.5-in. LG E1941 display connected to a machine running Windows 7. Presentation 17.0 software (Neurobehavioral Systems) was used to generate and display task stimuli and record participant responses from standard QWERTY-layout keyboards. The stimuli and procedure were adapted from those used by Nicholls et al. (2014). Targets and distractors were distributed across an invisible grid, 20 cells wide x 6 cells high, located in the centre of the display, subtending a visual angle of $28.98^{\circ}$ wide $\times 7.94^{\circ}$ high with grid elements evenly spaced approximately $1.50^{\circ}$ apart both horizontally and vertically. For target-absent trials, 60 cells were filled with identical upright equilateral black-outlined, white triangles $\left(0.44^{\circ}\right.$ wide and high) presented against a white background. Three triangles appeared in each of the 20 columns, with the vertical positioning of these triangles randomized across columns. An identical setup was used for target-present trials, except that one of the upright triangles was instead inverted (illustrated in Fig. 1). For each target-present trial, the inverted triangle appeared at a different location, and across the task appeared once at each of the 120 possible locations. Overall, there were 120 target-present trials and 24 target-absent trials, bringing the total number of trials for the task to 144 . Presentation order for the target-present and target-absent trials was randomized for each participant.

\section{Procedure}

Trials began with a central fixation cross, presented for 1,000 $\mathrm{ms}$. The search array then replaced the fixation cross and was presented for 2,000 ms, followed by a blank screen for 3,000 $\mathrm{ms}$. During the 5,000-ms window following the onset of the search array, participants were asked to indicate whether a 


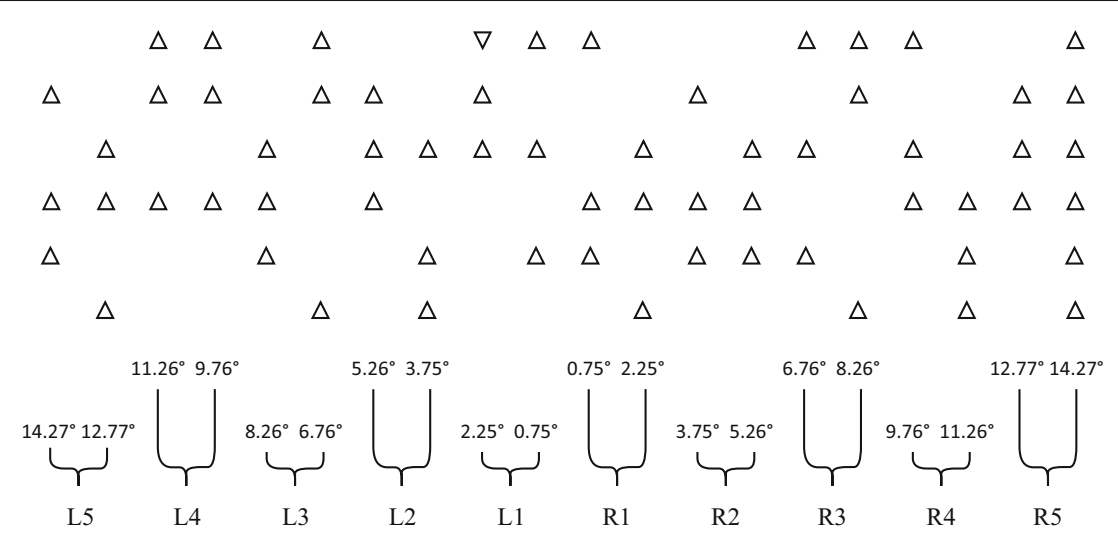

Fig. 1 An example of a target-present stimulus. Beneath are the eccentricities of each 'column' from the horizontal centre (i.e. $0^{\circ}$ ) and the ten position variables used for analysis. Only the triangles were presented during the task

target was present or absent as quickly and accurately as possible. Response mapping was counterbalanced across participants, with half using their left-hand to press the ' $Z$ ' key to indicate that a target was present and their right-hand to press the '/' key to indicate the absence of a target, whilst the remaining half used the reverse mapping. The trial immediately ended when a response was recorded, or when the 5,000-ms response window closed. In the latter 'time-out' situation, participants received a 1,000-ms visual prompt to respond faster and, rather than recording an erroneous response, the identical trial was presented again after all other trials had occurred, without the participant's knowledge (mean 'time-outs' per person $=1.81, S D=2.91$ ). Regardless of whether the trial ended with or without a response, a blank display was shown for $500 \mathrm{~ms}$, followed by the start of the next trial. This procedure continued until all trials for the task had been responded to. The 144 test trials were preceded by ten practice trials so that participants could familiarize themselves with the task and response mappings.

\section{Results}

\section{Overview and outlier screening}

Each participant's accuracy (i.e. proportion of trials answered correctly) was calculated separately for target-present and target-absent trials. Median reaction times (RTs) were calculated in the same manner but only for trials with correct responses. For target-present trials, multiple target locations were binned together to create ten different 'positions' that were entered into subsequent analyses (see Fig. 1) with the purpose of increasing statistical power and reliability of the data at each position by aggregating more trials at each spatial location. Specifically, neighbouring pairs of the columns used to generate the stimuli were binned together to create ten different positions labelled, from left to right, L5, L4, L3, L2, L1, $\mathrm{R} 1, \mathrm{R} 2, \mathrm{R} 3, \mathrm{R} 4$, and R5, with the digits representing distance from the screen centre and ' $L$ ' and ' $R$ ' signifying 'left' and 'right', respectively. The eccentricities associated with each position are given in Fig. 1.

Prior to conducting the main analyses, the dataset was screened for potential outliers. Mean accuracy for targetpresent and target-absent trials were calculated to be $77.41 \%$ $(S D=14.55 \%)$ and $89.31 \%(S D=16.20 \%)$, respectively, and overall mean task RT for target-present and target-absent trials were 1,228 $\mathrm{ms}(S D=237 \mathrm{~ms})$ and 2,133 $\mathrm{ms}(S D=366 \mathrm{~ms})$, respectively. Seven participants (all female) had target-present accuracy scores more than three SDs below the mean while eight participants (six female) had target-absent scores more than three SDs below the mean. One participant (female) had RTs more than three SDs below the mean while another two participants (both female) had RTs more than three SDs above the mean. Finally, a further four participants (one female) met multiple outlier criteria. In total, 22 participants were excluded from the main analyses.

\section{Target-present trials}

\section{Accuracy}

Task accuracy was examined using a 10 (position: withinsubjects) x 2 (sex: between-subjects) mixed-design analysis of variance (ANOVA) with the results summarised in Table 1 and means and 95\% confidence intervals for task accuracy separated by sex and position illustrated in Fig. 2. The analysis yielded separate main effects of position and sex, indicating that accuracy tended to be higher for more leftward target positions, and that males showed higher overall accuracy compared to females. A small but significant interaction between position and sex was also reported.

Polynomial contrasts were examined to better understand the effects involving target position. A linear trend was found for target position, showing that accuracy was greatest in more leftward locations and diminished as the target appeared more rightward. The interaction of the linear trend with sex 
Table 1 Results of two mixed-design ANOVAs separately analysing accuracy and reaction time as a function of participant sex and target position for target-present trials

\begin{tabular}{|c|c|c|c|c|c|c|c|}
\hline \multirow{2}{*}{ Comparison } & & \multicolumn{3}{|c|}{ Accuracy } & \multicolumn{3}{|c|}{ Reaction time } \\
\hline & & $\mathrm{F}$ & Sig. & $\eta_{\mathrm{p}}^{2}$ & $\mathrm{~F}$ & Sig. & $\eta_{\mathrm{p}}^{2}$ \\
\hline \multicolumn{2}{|c|}{ Within-subjects effects ${ }^{\dagger}$} & \multicolumn{3}{|c|}{$d f=8,4121$} & \multicolumn{3}{|c|}{$d f=6,3056$} \\
\hline \multicolumn{2}{|l|}{ Position } & 6.28 & $\leq .001 * * *$ & .012 & 147.39 & $\leq .001 * * *$ & .228 \\
\hline \multicolumn{2}{|l|}{ Position * Sex } & 1.97 & $.047 *$ & .004 & 1.23 & .28 & .002 \\
\hline \multicolumn{2}{|c|}{ Between-subjects effects } & \multicolumn{3}{|c|}{$d f=1,515$} & \multicolumn{3}{|c|}{$d f=1,499$} \\
\hline \multicolumn{2}{|l|}{ Sex } & 29.32 & $\leq .001 * * *$ & .054 & 9.02 & $\leq .01 * *$ & .018 \\
\hline \multicolumn{2}{|c|}{ Within-subjects contrasts } & \multicolumn{3}{|c|}{$d f=1,515$} & \multicolumn{3}{|c|}{$d f=1,499$} \\
\hline \multirow[t]{6}{*}{ Position } & Linear & 29.12 & $\leq .001 * * *$ & .054 & 114.81 & $\leq .001 * * *$ & .187 \\
\hline & Quadratic & 3.02 & .08 & .006 & 676.89 & $\leq .001 * * *$ & .576 \\
\hline & Cubic & 0.10 & .75 & .000 & 24.52 & $\leq .001 * * *$ & .047 \\
\hline & Order4 & - & - & - & 72.65 & $\leq .001 * * *$ & .127 \\
\hline & Order5 & - & - & - & 11.39 & $\leq .001 * * *$ & .022 \\
\hline & Order6 & - & - & - & 0.04 & .84 & .000 \\
\hline \multirow[t]{2}{*}{ Position * Sex } & Linear & 3.42 & .06 & .007 & 2.35 & .13 & .005 \\
\hline & Quadratic & 1.20 & .27 & .002 & 1.33 & .25 & .003 \\
\hline
\end{tabular}

$* \mathrm{p}<.05, * * \mathrm{p}<.01, * * * \mathrm{p}<.001 \%$ Greenhouse-Geisser corrected

approached statistical significance, with the slope somewhat steeper for males compared to females. Follow-up analyses examining polynomial contrasts for the two sexes separately revealed that linear trends were apparent for both males, $F(1$, $\left.148)=25.13, p<.001, \eta_{\mathrm{p}}{ }^{2}=.15\right)$, and females, $F(1,367)=$ $9.86, p<.01, \eta_{\mathrm{p}}{ }^{2}=.03$, although the effect size was notably larger for male participants.

Given the significant interaction between sex and target position, and that examination of the data in Fig. 2 suggested that sex differences were apparent at some, but not other, target positions, post hoc independent samples t-tests (Welch's, given unequal sample sizes) using a Bonferroni correction were conducted to compare accuracy for the two sexes at each of the ten target positions, with the results outlined in Table 2. To augment interpretation of the comparisons, Bayes factors for each comparison were also calculated using a default Cauchy prior of 0.707 and descriptive interpretations associated with each Bayes factor as outlined by Jeffreys (1961), with this information included alongside the t-tests. Notably, all comparisons of the two sexes for the leftpositioned targets revealed 'decisive' levels of evidence for greater male accuracy whilst comparisons for the rightpositioned targets were mixed in outcome.

\section{Reaction time}

Target RTs were examined using an identical $10 \times 2$ mixeddesign ANOVA with the results presented alongside the accuracy data in Table 1. Means and 95\% confidence intervals for task accuracy separated by sex and position are illustrated in Fig. 2. Complementing the analyses examining task accuracy, main effects of target position and sex were both
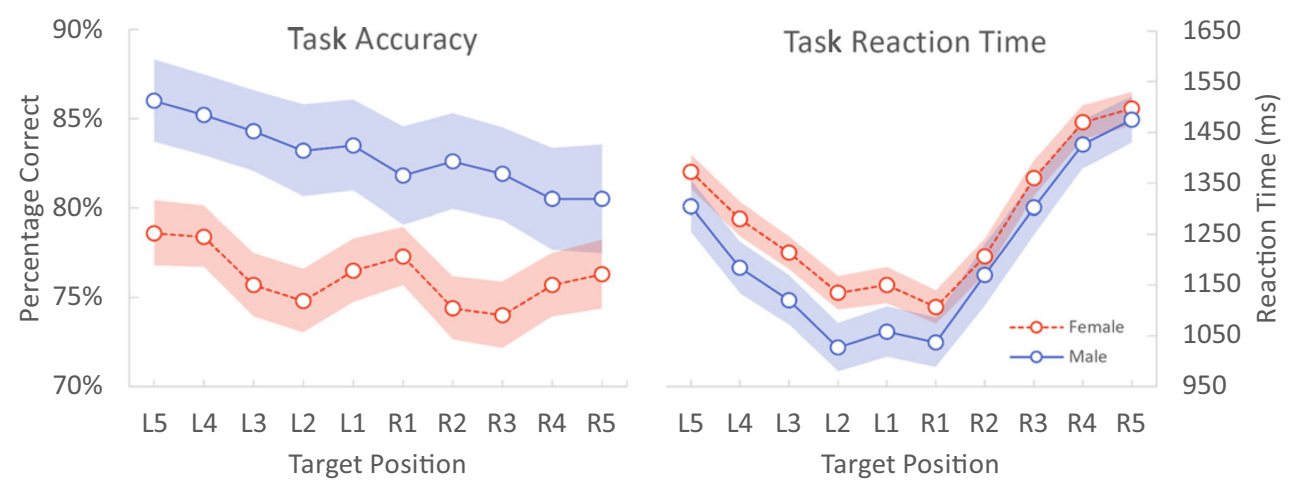

Fig. 2 Mean accuracy (left) and mean reaction time (right) as a function of target position and sex. Error bars are 95\% confidence intervals around the mean 
Table 2 Independent-sample Welch's t-tests comparing males and females on accuracy, reaction time, and Balanced Integration Scores at each target position for target-present trials

\begin{tabular}{|c|c|c|c|c|c|c|c|c|c|c|}
\hline & L5 & L4 & L3 & L2 & L1 & $\mathrm{R} 1$ & $\mathrm{R} 2$ & R3 & $\mathrm{R} 4$ & R5 \\
\hline \multicolumn{11}{|l|}{ Accuracy } \\
\hline (Welch's) $d f$ & 337 & 324 & 335 & 298 & 301 & 260 & 285 & 311 & 272 & 276 \\
\hline Welch's $t$ & 4.897 & 4.599 & 5.739 & 5.151 & 4.392 & 2.731 & 4.942 & 4.771 & 2.773 & 2.236 \\
\hline \multirow[t]{2}{*}{$p$} & $<.0001$ & $<.0001$ & $<.0001$ & $<.0001$ & $<.0001$ & 0.0067 & $<.0001$ & $<.0001$ & 0.0059 & 0.0262 \\
\hline & $* * *$ & $* * *$ & $* * *$ & $* * *$ & $* * *$ & n.s. & $* * *$ & $* * *$ & n.s. & n.s. \\
\hline$d$ & 0.435 & 0.415 & 0.51 & 0.482 & 0.409 & 0.272 & 0.471 & 0.438 & 0.27 & 0.216 \\
\hline$B F_{10}$ & 1513 & 651 & 51650 & 12799 & 510 & 4.67 & 7882 & 1736 & 4.44 & 1.18 \\
\hline $\begin{array}{l}B F_{10} \\
\quad \text { interpreta- } \\
\quad \text { tion }\end{array}$ & $\begin{array}{r}\text { Decisive } \\
\mathrm{H}_{1}\end{array}$ & $\begin{array}{r}\text { Decisive } \\
\mathrm{H}_{1}\end{array}$ & $\begin{array}{r}\text { Decisive } \\
\mathrm{H}_{1}\end{array}$ & $\begin{array}{r}\text { Decisive } \\
\mathrm{H}_{1}\end{array}$ & $\begin{array}{r}\text { Decisive } \\
\mathrm{H}_{1}\end{array}$ & $\begin{array}{r}\text { Substantial } \\
\mathrm{H}_{1}\end{array}$ & $\begin{array}{r}\text { Decisive } \\
\mathrm{H}_{1}\end{array}$ & $\begin{array}{r}\text { Decisive } \\
\mathrm{H}_{1}\end{array}$ & $\begin{array}{r}\text { Substantial } \\
\mathrm{H}_{1}\end{array}$ & $\begin{array}{r}\text { Anecdotal } \\
\mathrm{H}_{1}\end{array}$ \\
\hline \multicolumn{11}{|l|}{ Reaction time } \\
\hline (Welch's) $d f$ & 279 & 278 & 283 & 292 & 302 & 283 & 248 & 277 & 292 & 306 \\
\hline Welch's $t$ & -2.165 & -3.043 & -3.085 & -3.582 & -2.9 & -2.311 & -1.029 & -1.733 & -1.473 & -0.744 \\
\hline \multirow[t]{2}{*}{$P$} & 0.0312 & 0.0026 & 0.0022 & 0.0004 & 0.0040 & 0.0216 & 0.3045 & 0.0841 & 0.1418 & 0.4575 \\
\hline & n.s. & * & $*$ & $* *$ & $*$ & n.s. & n.s. & n.s. & n.s. & n.s. \\
\hline$D$ & 0.208 & 0.294 & 0.295 & 0.339 & 0.269 & 0.22 & 0.105 & 0.168 & 0.139 & 0.069 \\
\hline$B F_{10}$ & 0.98 & 8.78 & 9.07 & 36.3 & 4.36 & 1.28 & 0.19 & 0.455 & 0.289 & 0.137 \\
\hline $\begin{array}{l}B F_{10} \\
\quad \text { interpreta- } \\
\quad \text { tion }\end{array}$ & $\begin{array}{r}\text { Anecdotal } \\
\mathrm{H}_{0}\end{array}$ & $\begin{array}{r}\text { Substantial } \\
\mathrm{H}_{1}\end{array}$ & $\begin{array}{r}\text { Substantial } \\
\mathrm{H}_{1}\end{array}$ & $\begin{array}{r}\text { V. Strong } \\
\mathrm{H}_{1}\end{array}$ & $\begin{array}{r}\text { Substantial } \\
\mathrm{H}_{1}\end{array}$ & $\begin{array}{r}\text { Anecdotal } \\
\mathrm{H}_{1}\end{array}$ & $\begin{array}{r}\text { Substantial } \\
\mathrm{H}_{0}\end{array}$ & $\begin{array}{r}\text { Anecdotal } \\
\mathrm{H}_{0}\end{array}$ & $\begin{array}{r}\text { Substantial } \\
\mathrm{H}_{0}\end{array}$ & $\begin{array}{r}\text { Substantial } \\
\mathrm{H}_{0}\end{array}$ \\
\hline \multicolumn{11}{|c|}{ Balanced integration score (combined accuracy and reaction time) } \\
\hline (Welch's) $d f$ & 313 & 299 & 300 & 301 & 311 & 266 & 252 & 273 & 276 & 287 \\
\hline Welch's $t$ & 4.502 & 4.85 & 5.193 & 5.174 & 4.438 & 3.128 & 3.428 & 3.691 & 2.683 & 1.915 \\
\hline \multirow[t]{2}{*}{$p$} & $<.0001$ & $<.0001$ & $<.0001$ & $<.0001$ & $<.0001$ & 0.0020 & 0.0007 & 0.0003 & 0.0077 & 0.0564 \\
\hline & $* * *$ & $* * *$ & $* * *$ & $* * *$ & $* * *$ & $*$ & $* *$ & $* *$ & n.s. & n.s. \\
\hline$d$ & 0.412 & 0.454 & 0.485 & 0.482 & 0.407 & 0.307 & 0.347 & 0.36 & 0.26 & 0.182 \\
\hline$B F_{10}$ & 543 & 3416 & 14651 & 12851 & 481 & 12.9 & 48.6 & 76.4 & 3.36 & 0.583 \\
\hline $\begin{array}{l}B F_{10} \\
\quad \text { interpreta- } \\
\quad \text { tion }\end{array}$ & $\begin{array}{r}\text { Decisive } \\
\mathrm{H}_{1}\end{array}$ & $\begin{array}{r}\text { Decisive } \\
\mathrm{H}_{1}\end{array}$ & $\begin{array}{r}\text { Decisive } \\
\mathrm{H}_{1}\end{array}$ & $\begin{array}{r}\text { Decisive } \\
\mathrm{H}_{1}\end{array}$ & $\begin{array}{r}\text { Decisive } \\
\mathrm{H}_{1}\end{array}$ & Strong $\mathrm{H}_{1}$ & $\begin{array}{r}\text { V. Strong } \\
\mathrm{H}_{1}\end{array}$ & $\begin{array}{r}\text { V. Strong } \\
\mathrm{H}_{1}\end{array}$ & $\begin{array}{r}\text { Substantial } \\
\mathrm{H}_{1}\end{array}$ & $\begin{array}{r}\text { Anecdotal } \\
\mathrm{H}_{0}\end{array}$ \\
\hline
\end{tabular}

Note: Alpha values are adjusted using a Bonferroni correction. Adjusted traditional significance level cut-offs are as follows: $* p<.005, * * p<.001, * * *$ $p<.0001$, n.s. $=$ non-significant

reported, with RTs tending to be shorter for more central target positions and for the LVF compared to the RVF, and with male participants showing shorter RTs overall compared to female participants. In contrast to the accuracy data, however, the interaction between sex and target position was not statistically significant.

Post hoc polynomial contrasts revealed several significant trends involving target position. However, the quadratic trend was associated with the largest effect size, indicating that RT was fastest for centrally positioned targets and comparatively slower as targets were positioned further into the periphery. Additionally, of note was the linear trend, which had the nextlargest effect size and suggested that RTs were generally faster for left-positioned targets compared to right-positioned targets.
As with the accuracy data, visual inspection of the RT data in Fig. 2 was suggestive of alterations in the size of the sex difference across the visual field. Given this was our a priori prediction, we conducted independent-samples t-tests (using the same methodology as was used to examine the accuracy data) to investigate potential variations in the magnitude of sex differences for RT across the target positions. The results, outlined in Table 2, highlight a similar pattern of sex differences as was noted for the accuracy data. Sex differences, where present, were confined to targets positioned in the LVF (evidence from Bayesian t-tests ranged from anecdotal for $\mathrm{H} 0$ to 'very strong' for H1), whilst the target positions in the RVF were all statistically non-significant and yielded 'anecdotal' to 'substantial' levels of evidence in favour of the null hypothesis. 


\section{Combining accuracy and reaction time using balanced integration scores}

The accuracy and RT data both suggest that male participants tended to search more efficiently, particularly in the LVF, than female participants. However, due to individual differences in speed-accuracy trade-offs, it is possible that the male participants who showed the best accuracy did not also show the fastest RTs, and vice versa. Additionally, speed-accuracy trade-offs may have also varied depending on the position of the target. For example, a participant might be more careful searching a given location at the beginning of the trial (priority to accuracy), but become more haphazard in their search of remaining locations as the trial nears its expiry (priority to $\mathrm{RT})$.

We used the balanced-integration-score (BIS) approach to combine accuracy and RT into a single index as it has been shown to be relatively insensitive to speed-accuracy trade-offs compared to other methods (Liesefeld \& Janczyk, 2019). This method gives equal weighting to both accuracy and RT. The index is computed by standardising accuracy and RT across all participants and conditions (i.e. using Z-scores) and subtracting RT from accuracy. Using this approach, participants who achieved high accuracy at the expense of RT or fast RTs at the expense of accuracy, compared to other participants in the sample, will have BISs around zero. Participants with both high accuracy and fast RTs will have higher, positive BISs and participants with both low accuracy and slow RTs will have lower, negative BISs.

BIS was computed for each participant and the scores submitted to a $10 \times 2$ mixed-design ANOVA identical to the ones used previously to examine accuracy and RT, with the results outlined in Table 3. Means and 95\% confidence intervals for task accuracy separated by sex and position are illustrated in Fig. 3. Unlike the previous analyses on accuracy and RT, no main effect of position was found, suggesting that overall performance does not vary with target position. However, a main effect of sex was present, suggesting that male participants outperformed female participants on the task in general. An interaction between sex and position was also statistically significant, with Fig. 3 indicating that the size of the sex difference tended to be more pronounced the more leftward the target position.

A single significant polynomial contrast was found involving the interaction of sex with the linear trend across target position. Follow-up analysis revealed that the linear trend for target position was statistically significant for male participants, $F(1,146)=3.95, p=.05, \eta_{\mathrm{p}}^{2}=.03$, but was not statistically significant for female participants, $F(1,353)=1.39, p=$ $.24, \eta_{\mathrm{p}}{ }^{2}=.00$. Figure 3 illustrates how male participants identified targets more accurately and faster at more leftward positions, whereas the performance of female participants varied little as a function of target position. Table 2 summarises the $t-$
Table 3 Results of a mixed-design ANOVA analysing balanced integration score (BIS), a combined accuracy and reaction-time task performance index, as a function of participant sex and target position for targetpresent trials

\begin{tabular}{|c|c|c|c|c|}
\hline \multicolumn{2}{|l|}{ Comparison } & $\mathrm{F}$ & Sig. & $\eta_{\mathrm{p}}^{2}$ \\
\hline \multicolumn{2}{|c|}{ Within-subjects effects ${ }^{\dagger}$} & \multicolumn{3}{|c|}{$d f=6,3197$} \\
\hline \multicolumn{2}{|l|}{ Position } & 0.49 & .83 & .001 \\
\hline \multicolumn{2}{|l|}{ Position * Sex } & 2.17 & $.04 *$ & .004 \\
\hline \multicolumn{2}{|c|}{ Between-subjects effects } & \multicolumn{3}{|c|}{$d f=1,499$} \\
\hline \multicolumn{2}{|l|}{ Sex } & 23.08 & $\leq .001 * * *$ & .044 \\
\hline \multicolumn{2}{|c|}{ Within-subjects contrasts } & \multicolumn{3}{|c|}{$d f=1,499$} \\
\hline \multirow[t]{2}{*}{ Position } & Linear & 0.85 & .36 & .002 \\
\hline & Quadratic & 0.38 & .54 & .001 \\
\hline \multirow[t]{2}{*}{ Position * Sex } & Linear & 4.96 & $.03 *$ & .010 \\
\hline & Quadratic & 2.27 & .13 & .005 \\
\hline
\end{tabular}

$* \mathrm{p}<.05, * * * \mathrm{p}<.001$

$\dagger$ Greenhouse-Geisser corrected

test comparisons of the two sexes on the BIS for each of the ten target positions. All of the comparisons for the leftpositioned targets showed 'decisive' levels of evidence for males outperforming females, whilst comparisons for rightpositioned targets were mixed in level of support for this difference.

Finally, identical sets of analyses to those described above were conducted with the addition of 'key mapping' as a between-subjects variable. The aim in conducting these analyses was to check whether the effects associated with participant sex reported above were influenced by the Simon effect, a phenomenon in which participants perform better when target stimuli and the associated keyboard response are similarly lateralised (Simon \& Rudell, 1967). As roughly half of the participants were assigned the 'Z' key (left on the keyboard) to signify 'target present' and the remaining half used the ' $/$ key (right on the keyboard) instead, with the other key used to signify 'target absent', keyboard mapping could be easily

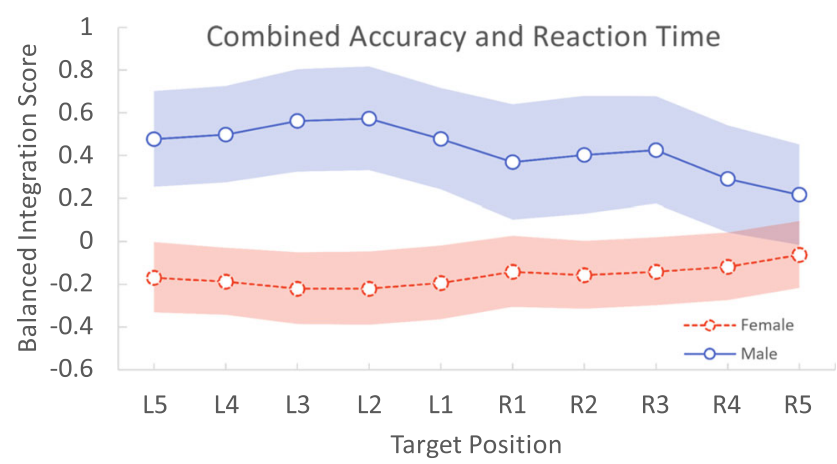

Fig. 3 Mean balanced integration score (BIS), a combined accuracy and reaction-time task performance index, as a function of target position and sex. Error bars are $95 \%$ confidence intervals around the mean 
controlled for with the addition of this variable. The expected Simon effect was present in these expanded analyses but, critically, the key mapping factor did not enter into a statistically significant interaction with participant sex in any of the analyses conduced. Given that the Simon effect was not of central interest and did not influence the outcomes of the present study, the results of these expanded analyses were relegated to the Online Supplementary Material.

\section{Target-absent trials}

Accuracy, RTs, and BIS for target-absent trials were also calculated to test for differences between sexes. Three independent-samples Welch's t-tests comparing male and female participants on each performance metric were conducted, with the results outlined in Table 4. No statistically significant differences were found for any comparison, suggesting that male and female participants performed comparably on target-absent trials.

\section{Discussion}

Sex differences in visuospatial ability have long been attributed to complementary differences in hemispheric asymmetry (Levy, 1972, 1978). However, a recent meta-analysis of studies examining both high-level visuospatial functioning and hemispheric asymmetry found little evidence for this association (Hirnstein et al., 2018). Whilst this work seemingly dispels the possibility of a direct link between sex differences and hemispheric asymmetry in high-level visuospatial functioning, little work has been done to test whether such links exist in relatively more perception-based tasks such as visual search. Given that stimuli qualitatively similar to visual search (i.e. line cancellation) are extensively used to examine differences in hemispheric asymmetry for healthy individuals and patients with unilateral hemispheric damage, it is interesting that visual search has rarely been used to explore sex differences in hemispheric asymmetry. To explore this possibility, the present study examined whether sex differences on a visual search task varied as a function of target location across the horizontal plane. The task was chosen for its ease with which stimuli could be adjusted to measure attention directed to different regions of the visual field.
Data obtained from the task indicate that the magnitude of the sex differences varied with the horizontal positioning of the target for target-present trials. Regarding task accuracy, sex and target position interacted, with t-tests comparing performance at each target position showing that the majority of sex differences existed in the LVF. Also of note was a nearstatistically significant interaction of sex with the linear trend for target position, with further investigation revealing a steeper linear trend for males compared to females. Similar patterns for RT were less clear as sex and target position did not interact significantly. However, a priori t-tests comparing performance at each target position provided a similar pattern to that found for the accuracy data, with males showing faster RTs for four of the five left-sided positions, and an absence of sex differences across all of the right-sided positions.

To accommodate potential speed-accuracy trade-offs, an additional analysis focused on the BIS obtained by combining accuracy and RT data, with this analysis revealing a similar pattern to that already outlined. A significant interaction between target position and sex was observed, with further analysis limiting this effect specifically to the interaction of the linear trend for target position with sex. Follow-up analyses indicated that the latter interaction was driven primarily by a linear trend for target position for males, but not females, with males displaying higher BIS scores (better performance) the more leftward the target position. This pattern of outcomes is in line with the suggestion that males display greater hemispheric asymmetry. Follow-up t-tests also suggested that sex differences for visual search performance were greatest in the LVF, with 'decisive' levels of evidence of sex differences for all five left-sided positions, and relatively lesser evidence for right-sided positions. Overall, the pattern of results corroborates the findings of Efron et al. (1987, 1990a), who used a different paradigm with fewer stimuli and shorter exposure durations, and therefore suggest that these effects are moderately robust and occur under varying conditions.

While the focus of the present study was on the interaction between sex and target position across the visual field, it must be noted that main effects of sex were found for accuracy, RT, and the balanced integration score, indicating that males outperformed female participants in terms of both speed and accuracy. This finding is in line with previous findings reported by Stoet (2011), but differs from the findings of Shaqiri et al.

Table 4 Accuracy, reaction time (RT), and balanced integration score (BIS) comparisons between male and female participants for target-absent trials

\begin{tabular}{lllll}
\hline & Male mean [95\% CI] & Female mean [95\% CI] & Welch's t & $p$ \\
\hline Accuracy & $91.50[89.79,93.21]$ & $90.94[89.76,92.13]$ & 0.52 & .60 \\
RT & $2172[2113,2230]$ & $2117[2080,2154]$ & 1.55 & .12 \\
BIS & $-0.07[-0.26,0.12]$ & $0.03[-0.10,0.16]$ & 0.84 & .05 \\
\hline
\end{tabular}


(2018) and Solianik et al. (2016). These discrepancies might be attributable to the different stimuli used across the different studies. For example, distinguishing a target ' $\mathrm{T}$ ' or line on the basis of both colour and orientation in the work by Stoet (2011) and Shaqiri et al. (2018) may involve a greater degree of cognitive processing than identifying a uniquely oriented triangle amongst identically oriented distractors. Differences in findings might also reflect the substantially greater statistical power in our study (with $\mathrm{N}=517$, compared to the nextlargest study of Shaqiri et al., 2018, with $\mathrm{N}=200$ ) and, thus, the ability to detect typically small sex differences (Hyde, 2005; Jäncke, 2018; Zell et al., 2015).

Several other notable findings were apparent in the data. Most prominent, perhaps, was the difference in performance trends between the accuracy and RT data collapsed across sex, with a small linear trend seen for accuracy and a strong quadratic trend present for RT. These trends might provide some clue as to the general 'strategy' taken by participants. If RT data is indicative of where participants searched, it could be interpreted that search began centrally, near fixation, and if the target was not identified there, search priority was then given to stimuli in the LVF over stimuli in the RVF, given the general advantage in RT for targets presented in the LVF. However, the accuracy data would suggest that this initial search near fixation was not as efficient as it could be, as accuracy was higher for targets presented to the left compared to those presented more centrally (e.g., for L1 vs. L5: $t(516)=$ $2.58, p=.01, d=.11$; see Fig. 2$)-$ perhaps indicating the beginning of a more systematic search. Interestingly, these trends tended to cancel out when examining the BIS, suggesting that speed-accuracy trade-offs varied depending on the position being searched, although the BIS did show a consistent improvement in performance for males as a function of more leftward presentation of the target. However, it is possible that different patterns may have been present for different task parameters. For example, shorter exposure durations (that limit eye-movements) are associated with higher accuracy near fixation (Cheal \& Lyon, 1989; Efron et al., 1987).

Whilst the present work sheds new light on an extensively used paradigm and highlights new sex differences in visual search performance, it is not without limitations. As this study was conducted using a convenience sample, certain demographic information was not obtained from participants that ought to be considered in future work. For example, estradiol and progesterone levels in women have been found to correlate with functional cerebral asymmetry, suggesting that the strength of any potential lateralisation effects is somewhat dependent on the participants' current phase in their menstrual cycles (for a review, see Hausmann, 2017). Given that the female participants likely varied in position in their monthly cycle, it is possible that our data oversimplifies the interaction between sex and target-position as some women may have shown less pronounced and others more pronounced hemispheric asymmetry than usual, as a function of the current phase of their cycle. It follows that future work should consider measuring this individual difference and using it as a covariate. Furthermore, experience playing videogames should also be considered in future work. Playing videogames, especially action-oriented games requiring quick reflexes, has been associated with improvements across numerous spatial tasks, including visual search (for a review, see Dye et al., 2009), though recent work suggests that the transfer of skills is small, at best (Sala et al., 2018). Despite more women reporting playing videogames in recent years, most gamers are male and male gamers tend to play for longer periods than females (Brand et al., 2017). This suggests that videogaming may influence males more than females and, thus, is another candidate variable to be considered as a covariate.

The sample tested, though large, was less well represented for males compared to females. However, as our results were far from marginal, and in line with earlier work (Efron et al., 1987, 1990a), we are disinclined to attribute the present findings to this disparity. Eye movements have also been linked to perceptual asymmetries in similar work (Nicholls et al., 2014). Thus, it would be beneficial to record eye movements in future work to help determine the degree to which differing patterns of performance between males and females are attributable to differing eye movements (and, potentially, search strategy) or search efficiency. That said, it should be noted that the sex differences in search performance we report are similar to those reported by Efron et al. (1987, 1990a) when using very short stimulus exposure durations (maximum $150 \mathrm{~ms}$ ) that might render eye movements useless. This suggests that our results were unlikely to be driven entirely by differences in search patterns or eye movements. Finally, though much can be gleaned from behavioural measures associated with hemispheric asymmetry (Behrmann et al., 2004; Saevarsson et al., 2009; Vangkilde \& Habekost, 2010), neuroimaging techniques could provide converging evidence on whether sex differences seen on the present visual search task are associated with differences in hemispheric asymmetry.

In summary, the present study provides evidence suggesting that the magnitude of sex differences varies as a function of target positioning along the horizontal plane. With respect to the current literature concerning sex differences in cognitive and spatial performance, and hemispheric asymmetry (Hirnstein et al., 2018), we caution against interpreting our results as evidence of a causal relationship between the two (i.e. that superior spatial performance is directly attributed to greater hemispheric asymmetry in males). However, these findings do suggest that such an option is possible and that studies to test for a causal link are warranted. Critically, the effects reported were sizable and thus could possibly add significant unexplained variance that might mask the effects of other independent variables, if not properly accounted for. 
Consequently, we suggest that researchers planning on using visual search tasks consider the effects of sex and laterality on task performance to ensure that any effects attributed to other factors can be tested independently and with precision.

Acknowledgements This research was supported by an Australian Research Council Discovery Project Grant (DP190103286) awarded to MTM and TAWV.

Author Contributions MCWE and TAWV conceived of the study. MCWE wrote the experimental software, oversaw data collection and performed statistical analyses. All authors contributed to interpreting the statistical analysis and writing the manuscript. All authors approved the final version of the manuscript prior to submission.

\section{Compliance with Ethical Standards}

Conflicts of interest The authors declare that they have no conflicts of interest.

\section{References}

Anstis, S. M. (1974). A chart demonstrating variations in acuity with retinal position. Vision Research, 14(7), 589-592. https://doi.org/ 10.1016/0042-6989(74)90049-2

Behrmann, M., Ebert, P., \& Black, S. E. (2004). Hemispatial neglect and visual search: A large scale analysis. Cortex, 40(2), 247-263. https:// doi.org/10.1016/S0010-9452(08)70120-5

Bowers, D., \& Heilman, K. M. (1980). Pseudoneglect: Effects of hemispace on a tactile line bisection task. Neuropsychologia, 18(4-5), 491-498. https://doi.org/10.1016/0028-3932(80)90151-7

Brand, J. E., Todhunter, S., \& Jervis, J. (2017). Digital Australia 2018. https:/www.igea.net/wp-content/uploads/2017/07/DigitalAustralia-2018-DA18-Final-1.pdf

Cheal, M., \& Lyon, D. (1989). Attention Effects on Form Discrimination at Different Eccentricities. The Quarterly Journal of Experimental Psychology Section A, 41(4), 719-746. https://doi.org/10.1080/ 14640748908402391

Dundas, E. M., Gastgeb, H., \& Strauss, M. S. (2012). Left visual field biases when infants process faces: a comparison of infants at highand low-risk for autism spectrum disorder. Journal of Autism and Developmental Disorders, 42(12), 2659-2668. https://doi.org/10. 1007/s10803-012-1523-y

Dye, M. W. G., Green, C. S., \& Bavelier, D. (2009). Increasing speed of processing with action video games. Current Directions in Psychological Science, 18(6), 321-326. https://doi.org/10.1111/j. 1467-8721.2009.01660.x

Efron, R., Yund, E. W., \& Nichols, D. R. (1987). Scanning the visual field without eye movements - A sex difference. Neuropsychologia, 25(4), 637-644. https://doi.org/10.1016/0028-3932(87)90054-6

Efron, R., Yund, E. W., \& Nichols, D. R. (1990a). Detectability as a function of target location: Effects of spatial configuration. Brain and Cognition, 12(1), 102-116. https://doi.org/10.1016/02782626(90)90007-B

Efron, R., Yund, E. W., \& Nichols, D. R. (1990b). Serial processing of visual spatial patterns in a search paradigm. Brain and Cognition, 12(1), 17-41. https://doi.org/10.1016/0278-2626(90)90002-6

Ellis, A. W., \& Brysbaert, M. (2010). Split fovea theory and the role of the two cerebral hemispheres in reading: A review of the evidence. Neuropsychologia, 48(2), 353-365. https://doi.org/10.1016/j. neuropsychologia.2009.08.021
Faul, F., Erdfelder, E., Lang, A.-G., \& Buchner, A. (2007). G*Power 3: A flexible statistical power analysis program for the social, behavioral, and biomedical sciences. Behavior Research Methods, 39(2), 175191. https://doi.org/10.3758/BF03193146

Ferber, S., \& Karnath, H. O. (2001). How to assess spatial neglect - Line bisection or cancellation tasks? Journal of Clinical and Experimental Neuropsychology, 23(5), 599-607. https://doi.org/ 10.1076/jcen.23.5.599.1243

Hahn, N., Jansen, P., \& Heil, M. (2010). Preschoolers' mental rotation of letters: Sex differences in hemispheric asymmetry. Cognitive Neuroscience, 1(4), 261-267. https://doi.org/10.1080/17588928. 2010.485248

Halpern, D. F. (2012). Empirical Evidence For Cognitive Sex Differences. In Sex differences in cognitive abilities (4th ed.). Psychology Press, Taylor and Francis Group.

Hattie, J. (2009). Visible Learning: A Synthesis of Over 800 MetaAnalyses Relating to Achievement. Routledge Ltd. https://doi.org/ $10.4324 / 9780203887332$

Hausmann, M. (2017). Why sex hormones matter for neuroscience: A very short review on sex, sex hormones, and functional brain asymmetries. Journal of Neuroscience Research, 95(1-2), 40-49. https://doi.org/10.1002/jnr.23857

Heilman, K. M. (1995). Attentional asymmetries. In R. J. Davidson \& K. Hugdahl (Eds.), Brain Asymmetry (pp. 217-234). MIT Press.

Heilman, K. M., Watson, R. T., \& Valenstein, E. (2003). Neglect and related disorders. In K. M. Heilman \& E. Valenstein (Eds.), Clinical Neuropsychology (pp. 279-336). Oxford University Press.

Hellige, J. B. (1993). Hemispheric Asymmetry: What's Right and What's Left (S. M. Kosslyn (ed.)). Harvard University Press.

Hirnstein, M., Hugdahl, K., \& Hausmann, M. (2018). Cognitive sex differences and hemispheric asymmetry: A critical review of 40 years of research. Laterality: Asymmetries of Body, Brain and Cognition, O(0), 1-49. https://doi.org/10.1080/1357650X.2018. 1497044

Holcombe, A. O., Nguyen, E. H. L., \& Goodbourn, P. T. (2017). Implied reading direction and prioritization of letter encoding. Journal of Experimental Psychology: General, 146(10), 1420-1437. https:// doi.org/10.1037/xge0000357

Hyde, J. S. (2005). The gender similarities hypothesis. American Psychologist, 60(6), 581-592. https://doi.org/10.1037/0003-066X. 60.6 .581

Jäncke, L. (2018). Sex/gender differences in cognition, neurophysiology, and neuroanatomy. F1000Research, 7, 805. https://doi.org/10. 12688/f1000research.13917.1

Jeffreys, H. (1961). Theory of Probability (3rd Ed.). Oxford University Press.

Jewell, G., \& McCourt, M. E. (2000). Pseudoneglect: a review and metaanalysis of performance factors in line bisection tasks. Neuropsychologia, 38(1), 93-110. https://doi.org/10.1016/S00283932(99)00045-7

Jordan, T. R., Patching, G. R., \& Thomas, S. M. (2003). Assessing the role of hemispheric specialisation, serial-position processing, and retinal eccentricity in lateralised word recognition. Cognitive Neuropsychology, 20(1), 49-71. https://doi.org/10.1080/ 02643290244000185

Levy, J. (1972). Lateral specialization of the human brain: Behavioral manifestations and possible evolutionary basis. In J. A. Kiger (Ed.), The Biology of Behavior: Proceedings of the thirty-second annual biology collquium. Oregon State University Press.

Levy, J. (1978). Lateral differences in the human brain in cognition and behavioral control. In P. Buser \& A. Rougeul-Buser (Eds.), Cerebral correlates of conscious experimence (pp. 285-298). North Holland Publishing Company.

Liesefeld, H. R., \& Janczyk, M. (2019). Combining speed and accuracy to control for speed-accuracy trade-offs(?). Behavior Research Methods, 51(1), 40-60. https://doi.org/10.3758/s13428-018-1076-x 
Nicholls, M. E. R., Bradshaw, J. L., \& Mattingley, J. B. (1999). Freeviewing perceptual asymmetries for the judgement of brightness, numerosity and size. Neuropsychologia, 37(3), 307-314. https:// doi.org/10.1016/S0028-3932(98)00074-8

Nicholls, M. E. R., Hobson, A., Petty, J., Churches, O., \& Thomas, N. A. (2017). The effect of cerebral asymmetries and eye scanning on pseudoneglect for a visual search task. Brain and Cognition, 111, 134-143. https://doi.org/10.1016/j.bandc.2016.11.006

Nicholls, M. E. R., Roden, S., Thomas, N. A, Loetscher, T., Spence, C. J., \& Forte, J. D. (2014). Close to me: the effect of asymmetrical environments on spatial attention. In Ergonomics (Vol. 57, Issue February, pp. 876-885). Taylor \& Francis. https://doi.org/10.1080/ 00140139.2014 .899633

Ocklenburg, S., \& Güntürkün, O. (2017). The Lateralized Brain: The Neuroscience and Evolution of Hemispheric Asymmetries. Academic Press.

Oldfield, R. C. (1971). The assessment and analysis of handedness: The Edinburgh inventory. Neuropsychologia, 9(1), 97-113. https://doi. org/10.1016/0028-3932(71)90067-4

Poynter, W., \& Roberts, C. (2012). Hemispheric asymmetries in visual search. Laterality, 17(6), 711-726. https://doi.org/10.1080/ 1357650X.2011.626558

Ransley, K., Goodbourn, P. T., Nguyen, E. H. L., Moustafa, A. A., \& Holcombe, A. O. (2018). Reading Direction Influences Lateral Biases in Letter Processing. Journal of Experimental Psychology: Learning Memory and Cognition, 44(10), 1678-1686. https://doi. org $/ 10.1037 / x \operatorname{lm} 0000540$

Rilea, S. L. (2008). Sex and hemisphere differences when mentally rotating meaningful and meaningless stimuli. Laterality, 13(3), 217-233. https://doi.org/10.1080/13576500701809846

Rossion, B., Dricot, L., Devolder, A., Bodart, J.-M., Crommelinck, M., Gelder, B. de, \& Zoontjes, R. (2000). Hemispheric Asymmetries for Whole-Based and Part-Based Face Processing in the Human Fusiform Gyrus. Journal of Cognitive Neuroscience, 12(5), 793 802. https://doi.org/10.1162/089892900562606

Saevarsson, S., Kristjánsson, Á., Hildebrandt, H., \& Halsband, U. (2009). Prism adaptation improves visual search in hemispatial neglect. Neuropsychologia, 47(3), 717-725. https://doi.org/10.1016/j. neuropsychologia.2008.11.026

Sala, G., Tatlidil, K. S., \& Gobet, F. (2018). Video game training does not enhance cognitive ability: A comprehensive meta-analytic investigation. Psychological Bulletin, 144(2), 111-139. https://doi.org/10. 1037/bul0000139

Shaqiri, A., Roinishvili, M., Grzeczkowski, L., Chkonia, E., Pilz, K., Mohr, C., Brand, A., Kunchulia, M., \& Herzog, M. H. (2018). Sex-related differences in vision are heterogeneous. Scientific Reports, 8(1), 1-10. https://doi.org/10.1038/s41598-018-25298-8

Simon, J. R., \& Rudell, A. P. (1967). Auditory S-R compatibility: The effect of an irrelevant cue on information processing. Journal of
Applied Psychology, 51(3), 300-304. https://doi.org/10.1037/ h0020586

Solianik, R., Brazaitis, M., \& Skurvydas, A. (2016). Sex-related differences in attention and memory. Medicina (Lithuania), 52(6), 372377. https://doi.org/10.1016/j.medici.2016.11.007

Stoet, G. (2011). Sex differences in search and gathering skills. Evolution and Human Behavior, 32(6), 416-422. https://doi.org/10.1016/j. evolhumbehav.2011.03.001

Treisman, A. M., \& Gelade, G. (1980). A feature-integration theory of attention. Cognitive Psychology, 12(1), 97-136. https://doi.org/10. 1016/0010-0285(80)90005-5

Vangkilde, S., \& Habekost, T. (2010). Finding Wally: Prism adaptation improves visual search in chronic neglect. Neuropsychologia, 48(7), 1994-2004. https://doi.org/10.1016/j.neuropsychologia.2010.03. 020

Vanston, J. E., \& Strother, L. (2017). Sex differences in the human visual system. Journal of Neuroscience Research, 95(1-2), 617-625. https://doi.org/10.1002/jnr.23895

Verleger, R., \& Śmigasiewicz, K. (2015). Consciousness wanted, attention found: Reasons for the advantage of the left visual field in identifying T2 among rapidly presented series. Consciousness and Cognition, 35, 260-273. https://doi.org/10.1016/j.concog.2015.02. 013

Wolfe, J. M. (1994). Guided Search 2.0: A revised model of visual search. Psychonomic Bulletin \& Review, 1(2), 202-238. https://doi.org/10. 3758/BF03200774

Yund, E. W., Efron, R., \& Nichols, D. R. (1990a). Detectability as a function of spatial location: Effects of selective attention. Brain and Cognition, 12(1), 42-54. https://doi.org/10.1016/02782626(90)90003-7

Yund, E. W., Efron, R., \& Nichols, D. R. (1990b). Detectability gradients as a function of target location. Brain and Cognition, 12(1), 1-16. https://doi.org/10.1016/0278-2626(90)90001-5

Zell, E., Krizan, Z., \& Teeter, S. R. (2015). Evaluating gender similarities and differences using metasynthesis. American Psychologist, 70(1), 10-20. https://doi.org/10.1037/a0038208

Open Practices Statement The dataset used to conduct the analyses for this study is available at the University of Western Australia's Research Repository: https://doi.org/10.26182/5e575c32dd0b6. The computerised behavioural task is available on reasonable request by emailing the corresponding author. The experiment was not preregistered.

Publisher's note Springer Nature remains neutral with regard to jurisdictional claims in published maps and institutional affiliations. 